\title{
Crystal Structure of a Deacylation-defective Mutant of Penicillin-binding Protein 5 at 2.3-Å Resolution*
}

Received for publication, May 24, 2000, and in revised form, August 15, 2000

Published, JBC Papers in Press, August 30, 2000, DOI 10.1074/jbc.M004471200

\author{
Christopher Davies\$, Stephen W. White§n, and Robert A. Nicholas|*** \\ From the $\ddagger$ School of Biological Sciences, University of Sussex, Falmer, Brighton BN1 9QG, United Kingdom, \\ $\S$ Department of Structural Biology, St. Jude Children's Research Hospital, Memphis, Tennessee 38105, \\ १Department of Biochemistry, University of Tennessee, Memphis, Tennessee 38163, and |Department of Pharmacology, \\ University of North Carolina at Chapel Hill, Chapel Hill, North Carolina 27599-7365
}

\begin{abstract}
Penicillin-binding protein 5 (PBP 5) of Escherichia coli functions as a D-alanine carboxypeptidase, cleaving the $\mathrm{C}$-terminal D-alanine residue from cell wall peptides. Like all PBPs, PBP 5 forms a covalent acyl-enzyme complex with $\beta$-lactam antibiotics; however, PBP 5 is distinguished by its high rate of deacylation of the acyl-enzyme complex $\left(t_{1 / 2} \sim 9 \mathrm{~min}\right)$. A Gly-105 $\rightarrow$ Asp mutation in PBP 5 markedly impairs this $\beta$-lactamase activity (deacylation), with only minor effects on acylation, and promotes accumulation of a covalent complex with peptide substrates. To gain further insight into the catalytic mechanism of PBP 5, we determined the three-dimensional structure of the G105D mutant form of soluble PBP 5 (termed sPBP 5') at $2.3 \AA$ resolution. The structure is composed of two domains, a penicillin binding domain with a striking similarity to Class A $\beta$-lactamases (TEM-1-like) and a domain of unknown function. In addition, the penicillin-binding domain contains an active site loop spatially equivalent to the $\Omega$ loop of $\beta$-lactamases. In $\beta$-lactamases, the $\Omega$ loop contains two amino acids involved in catalyzing deacylation. This similarity may explain the high $\beta$-lactamase activity of wild-type PBP 5. Because of the low rate of deacylation of the G105D mutant, visualization of peptide substrates bound to the active site may be possible.
\end{abstract}

Penicillin and other $\beta$-lactam antibiotics exert their lethal effect by inhibiting the proteins that synthesize bacterial cell wall peptidoglycan (1). These proteins, known as penicillinbinding proteins or $\mathrm{PBPs}_{\mathrm{s}}{ }^{1}$ utilize lipid-linked disaccharide peptide substrates to catalyze both the polymerization of glycan chains (transglycosylation) and cross-linking of peptide chains (transpeptidation) during cell wall synthesis. In the latter reaction, a serine residue on the $\mathrm{PBP}$ reacts with the acyl-D-Ala-D-Ala $\mathrm{C}$ terminus of the peptide chain to form a transient acyl-enzyme complex, releasing the C-terminal Dalanine residue. This complex reacts with an amino group from

* The costs of publication of this article were defrayed in part by the payment of page charges. This article must therefore be hereby marked "advertisement" in accordance with 18 U.S.C. Section 1734 solely to indicate this fact.

The atomic coordinates and structure factors (code 1hd8) have been deposited in the Protein Data Bank, Research Collaboratory for Structural Bioinformatics, Rutgers University, New Brunswick, NJ (http://www.rcsb.org/).

** To whom correspondence should be addressed: Dept. of Pharmacology, CB\#7365 Mary Ellen Jones Bldg., Chapel Hill, NC 27599-7365. E-mail: nicholas@med.unc.edu.

${ }^{1}$ The abbreviations used are: PBP, penicillin-binding protein; sPBP, soluble PBP; CPase, carboxypeptidase; MIR, multiple isomorphous replacement; r.m.s., root mean square. another peptide chain to form a cross-link, which is crucial to the integrity and rigidity of the cell wall. An additional activity catalyzed by some PBPs, carboxypeptidation, occurs when the acyl-enzyme complex reacts with water. Penicillin and other $\beta$-lactam antibiotics mimic the structure of the acyl-D-Ala-D-Ala $\mathrm{C}$ terminus of the peptide chain (2) and react with PBPs to form an acyl-enzyme complex. Unlike the transient nature of the PBP-peptide complex, the acyl-enzyme complex formed between PBPs and $\beta$-lactam antibiotics is much more stable and results in prolonged inhibition of the enzyme.

In Escherichia coli, at least 10 PBPs have been identified. These PBPs can be split into two classes: the high molecular mass PBPs (PBPs 1A, 1B, 1C, 2, and 3) and the low molecular mass PBPs (PBPs 4, 5, 6, 6b, and 7) (3). High molecular mass PBPs are essential for cell viability and are involved in the physiological processes of cell elongation, cell division, and the maintenance of cell shape (4). The role of low molecular mass PBPs in bacterial physiology is less clear. These PBPs catalyze D,D-endopeptidase or D,D-carboxypeptidase activity both in vitro and in vivo $(5,6)$, but although they account for $>90 \%$ of the amount of penicillin $\mathrm{G}$ bound to membranes, they are not essential for cell viability (7). However, a recent study utilizing strains with multiple deletions of low molecular mass PBPs revealed a role for PBP 5 in the proper synthesis of peptidoglycan (8).

Production of a $\beta$-lactamase is the most common mechanism by which bacteria become resistant to $\beta$-lactam antibiotics (9). The initial step of the reaction catalyzed by these enzymes is analogous to that mediated by PBPs, but instead of accumulating a stable acyl-enzyme complex, $\beta$-lactamases destroy the antibiotic by rapidly hydrolyzing the acyl-enzyme bond. Analysis of the sequences and crystal structures of PBPs and Class $\mathrm{A}$ and class $\mathrm{C} \beta$-lactamases reveals strong structural similarities in the two classes of penicillin-interacting proteins (1012). The hallmark of all active site serine-based penicillininteracting proteins is the presence of three well conserved motifs in the active site (13). These motifs are the SXXK tetrad containing the active site serine residue, the $(\mathrm{S} / \mathrm{Y}) X \mathrm{~N}$ triad, and the $\mathrm{KT}(\mathrm{S}) \mathrm{G}$ triad.

The reaction mechanism of the interaction of $\beta$-lactam antibiotics with PBPs and $\beta$-lactamases is represented schematically by the following three-step model.

$$
E+S \underset{k_{-1}}{\stackrel{k_{+1}}{\rightleftharpoons}} E \cdot S \stackrel{k_{2}}{\longrightarrow} E-S^{*} \stackrel{k_{3}}{\longrightarrow} E+P
$$

where $E$ is the PBP or $\beta$-lactamase, $S$ is a $\beta$-lactam antibiotic, $E \cdot S$ is the Michaelis complex, $E$ - $S^{*}$ is the covalent acyl-enzyme complex, and $P$ is the inactive degradation product $(14,15)$. In PBPs, $k_{3}$ is usually quite small compared with $k_{2}$, leading to 
accumulation of the inactive acylated enzyme. For $\beta$-lactamases, both the $k_{2}$ and $k_{3}$ rate constants are very large, and the $\beta$-lactam antibiotic is hydrolyzed.

In contrast to most other PBPs, $E$. coli PBP 5 is distinguished by its high $\beta$-lactamase activity, with $\mathrm{k}_{3} \approx 0.07 \mathrm{~s}^{-1}\left(t_{1 / 2}<10\right.$ min) for the penicilloyl-PBP 5 complex. A mutant PBP 5, PBP 5-G105D (termed PBP 5'), shows near normal acylation rates with penicillin $\mathrm{G}$ but displays a 30 -fold decrease in the rate of deacylation $(16,17)$. Additionally, the mutant protein forms a stable covalent complex with the depsipeptide substrate, $N, N^{\prime}$ diacetyl-L-lysyl-D-alanyl-D-lactate, which is not observed with the wild-type protein (16). The phenotype of PBP $5^{\prime}$ is similar to that of wild-type PBP 5 in which Cys- 115 has been modified with sulfhydryl reagents, causing a larger effect on deacylation than acylation (18). However, since the mutants PBP 5-C115A and PBP 5-C115S both display wild-type levels of D-alanine carboxypeptidase (CPase) and penicillin binding activities, this residue does not appear to be directly involved in the catalytic mechanism of the enzyme (17). Subsequent attempts to identify residues involved in deacylation of PBP 5 have been unsuccessful (19).

In an effort to understand the molecular mechanism of deacylation of PBP 5 and to define further the interactions of $\beta$-lactam antibiotics and peptide substrates with PBPs, we have solved the structure of the G105D mutant of PBP 5 at 2.3- $\AA$ resolution, the first structure for PBP 5 . It reveals the closest similarity yet identified between a PBP-type enzyme and a Class A $\beta$-lactamase, which may explain the relatively high $\beta$-lactamase activity of PBP 5 .

\section{MATERIALS AND METHODS}

Protein Purification and Crystallization-Construction of the expression plasmid encoding sPBP $5^{\prime}$ has been described $(17,20)$. The gene encoding the G105D mutant form of PBP 5 missing its last 17 codons (plus six extra codons added during plasmid construction) was cloned into the PstI-HindIII restriction sites of pBR322. Overnight cultures of MC1061 harboring the expression plasmid were subjected to osmotic shock (21), and sPBP $5^{\prime}$ was purified from the shock fluid by ampicillin affinity chromatography exactly as described (17). The purified protein was dialyzed exhaustively against $20 \mathrm{~mm}$ Tris $\cdot \mathrm{HCl}, 150 \mathrm{~mm} \mathrm{NaCl}, 10$ mM 2-mercaptoethanol, $0.2 \% \mathrm{NaN}_{3}, \mathrm{pH} 7.5$ and concentrated to $6-8 \mathrm{mg}$ $\mathrm{ml}^{-1}$. SDS-polyacrylamide electrophoresis indicated that the protein was $>98 \%$ pure with very little of the characteristic $30-$ and $10-\mathrm{kDa}$ breakdown products (22).

Crystallization conditions were similar to those described previously (23). Crystals were grown by vapor diffusion in $20 \%$ polyethylene glycol
4000, $50 \mathrm{~mm}$ Tris $\cdot \mathrm{HCl}, \mathrm{pH} 7.0,0.2 \% \mathrm{NaN}_{3}$. Crystals of bullet-shaped morphology formed at $18{ }^{\circ} \mathrm{C}$ within $1-2$ days. The crystals belong to space group P32 with cell dimensions $a=50.83 \AA$ and $b=c=140.29$ $\AA$. There is one molecule in the asymmetric unit.

Data Collection-In all cases crystals were mounted in quartz capillaries, and data were collected at room temperature by the standard oscillation method. The initial native data set was collected using a Rigaku RAXIS-II image plate system (Molecular Structures Corp., The Woodlands, TX) mounted on a Rigaku RU-300 x-ray generator operating at $40 \mathrm{kV}$ and $80 \mathrm{~mA}$ and fitted with a graphite monochromator. The crystal-to-plate distance was $130 \mathrm{~mm}$, the oscillation angle was $3^{\circ}$, and the exposure time was $30 \mathrm{~min}$ frame $\mathrm{e}^{-1}$. A total of $84^{\circ}$ of data was collected.

Derivative diffraction data were collected using a DIP 2030H image plate detector mounted on a Nonius FR391 rotating anode x-ray generator fitted with MacScience focusing mirrors (Nonius B. V., Delft, The Netherlands) and operating at $40 \mathrm{kV}$ and $100 \mathrm{~mA}$. Data were collected at a crystal-to-plate distance of $150 \mathrm{~mm}$, with an oscillation angle of $1^{\circ}$ and an exposure time of $18 \mathrm{~min}$ frame $\mathrm{e}^{-1}$. Rotation ranges of $60-75^{\circ}$ were sufficient to collect essentially complete data sets.

For model refinement purposes a high resolution data set was collected, where the crystal-to-plate distance was $150 \mathrm{~mm}$, the oscillation angle was $1^{\circ}$, and the exposure time was 12 min frame $^{-1}$. To ensure a high redundancy of data, the crystal was rotated through a total of $90^{\circ}$. In all cases the oscillation data were processed using HKL (24). In the case of derivative data, the Friedel pairs were not merged in order to retain the anomalous signal.

Phasing-A search for derivatives was made by soaking crystals in solutions of heavy atom compounds in stabilizing buffer (20\% polyethylene glycol 4000 in $100 \mathrm{~mm}$ Tris $\cdot \mathrm{HCl}, \mathrm{pH}$ 7.0). Soaks in various mercury and platinum compounds yielded several promising derivatives, as judged by difference Patterson maps. In each case heavy atom positions

TABLE I

Data collection statistics

$\mathrm{Hg}(\mathrm{CN})_{2}$, mercury (II) cyanide; PIP, di- $\mu$-iodobis(ethylenediamine) di-platinum nitrate.

\begin{tabular}{|c|c|c|c|c|}
\hline & Native 1 & Native 2 & $\mathrm{Hg}(\mathrm{CN})_{2}$ & PIP \\
\hline Molarity (mM) & & & 1 & 10/sat. \\
\hline Length of soak & & & $2 \mathrm{~d}$ & $1 \mathrm{~h}$ \\
\hline Temperature (K) & 298 & 298 & 298 & 298 \\
\hline Reflections (unique) & 13776 & 17239 & 13482 & 9584 \\
\hline Reflections (measured) & 37497 & 43406 & 34123 & 18341 \\
\hline Completeness (\%) & 98.0 & 94.8 & 95.6 & 95.9 \\
\hline Resolution (凡) & 2.5 & 2.3 & 2.5 & 2.8 \\
\hline$R_{\mathrm{SYM}}{ }^{a}$ & 7.9 & 6.9 & 7.4 & 9.1 \\
\hline
\end{tabular}

TABLE II

Phasing statistics

Derivative abbreviations are as in Table I.

\begin{tabular}{|c|c|c|c|c|}
\hline \multirow{2}{*}{$\begin{array}{l}\text { Derivative } \\
\text { Type of data }\end{array}$} & \multicolumn{2}{|c|}{$\mathrm{Hg}(\mathrm{CN})_{2}$} & \multicolumn{2}{|c|}{ PIP } \\
\hline & Isomorphous & Anomalous & Isomorphous & Anomalous \\
\hline Resolution & 2.5 & 2.5 & 2.8 & 2.8 \\
\hline No. of reflections & 7246 & 5863 & 5182 & 4003 \\
\hline$R_{\mathrm{MERGE}}{ }^{a}$ & 20.3 & & 6.5 & \\
\hline Phasing power ${ }^{b}$ & 1.28 & 0.91 & 1.43 & 0.70 \\
\hline$R_{\text {CULLIS }}{ }^{c}$ & 0.849 & 0.946 & 0.777 & 0.971 \\
\hline$R_{\text {KRAUT }}{ }^{d}$ & 0.203 & 0.514 & 0.132 & 0.577 \\
\hline \multicolumn{5}{|l|}{ Overall figure of merit: } \\
\hline Before solvent flattening & & 0.244 & & \\
\hline After solvent flattening & & 0.933 & & \\
\hline
\end{tabular}

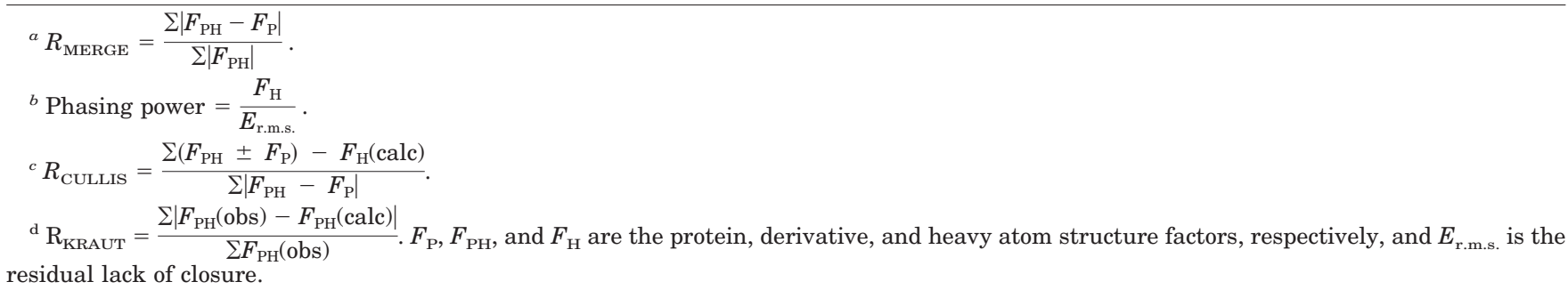




\section{A}
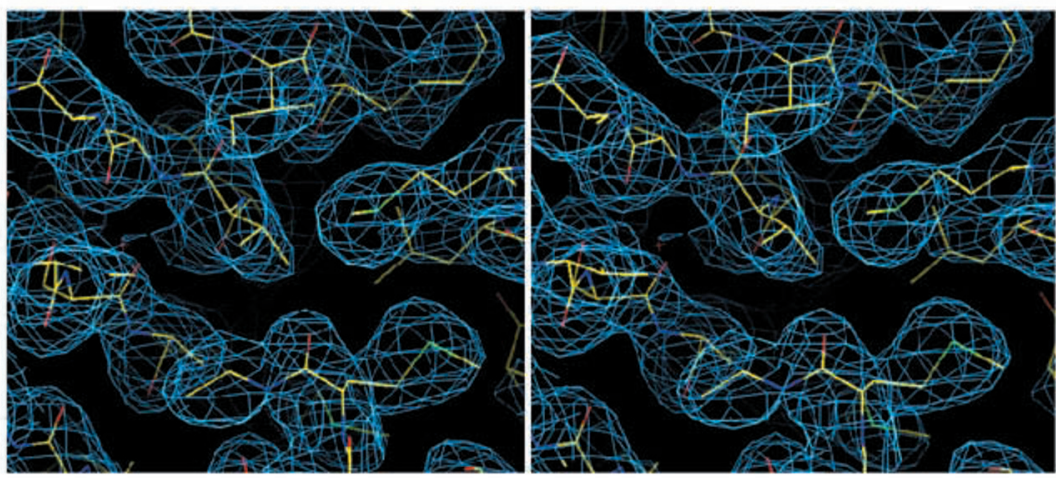

FIG. 1. Stereoviews of the electron density maps of $\mathbf{s P B P} 5^{\prime} . A$, the solvent-flattened MIR map at 2.5-A resolution. $B$, the $2 / F_{o}-F_{c}$ calculated phased map generated from the final refined coordinates at $2.3 \mathrm{~A}$. The region shown is a portion of the hydrophobic core in domain 1 . Both maps are contoured at $1 \sigma$ and are displayed using $\mathrm{O}(29)$.

\section{MIR Map}
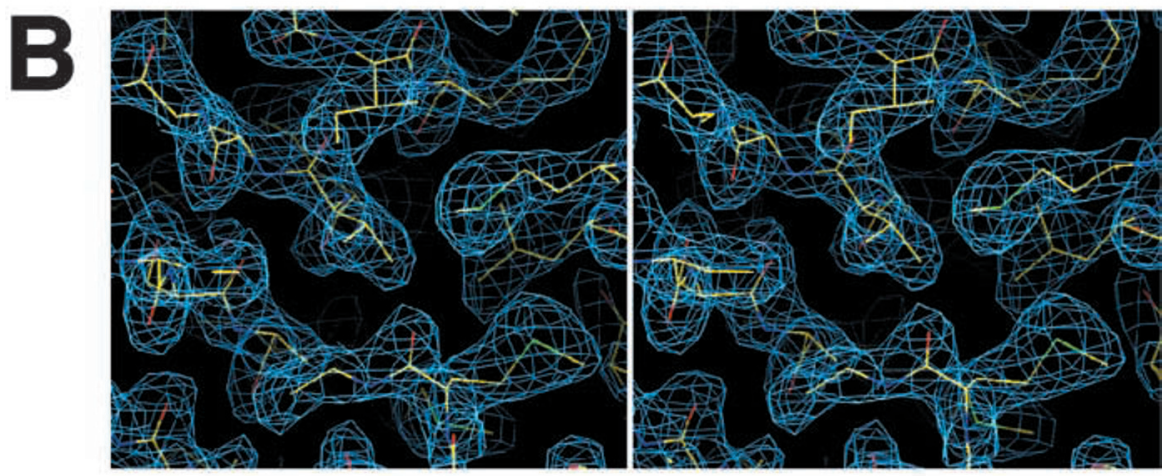

\section{2|Fo-Fcl map}

were determined and refined, and the data were used in trial phasing. These calculations showed that two derivatives, mercury cyanide $\left(\mathrm{HgCN}_{2}\right)$ and di- $\mu$-iodobis-(ethylenediamine) di-platinum nitrate (PIP), were of the best quality and sufficient to solve the structure. All calculations to this point were performed using PHASES (25). At this stage the derivative and native data were merged using SCALEIT (26), and the final phasing calculation was performed using SHARP (27) and included anomalous data. The resulting multiple isomorphous replacement (MIR) phases were improved by solvent flattening using SOLOMON (28) with an estimated solvent content of $46 \%$.

Model Building and Refinement-An MIR electron density map (Fig. $1 B$ ) was calculated using CCP4 programs (26) and displayed using the program $O$ (29). The map was of excellent quality and was easily interpreted to build the entire structure, including side chains, with the exception of residues 1-3 and 74-90. The model was then refined by alternating rounds of XPLOR and manual revision using $\mathrm{O}$. In later rounds water molecules were included. The final round of refinement was performed using REFMAC (26). The stereochemistry of the final model was evaluated using PROCHECK (30). The numbering of the final model corresponds to the sequence of the mature processed protein.

\section{RESULTS}

\section{Structure Determination}

The structure of sPBP 5' was determined by MIR with anomalous scattering. The data collection and phasing statistics are shown in Tables I and II, and portions of both the MIR and final $2 / F_{o}-F_{c}$ electron density maps are shown in Fig. 1 . The final structure has an $R$ factor of $19.6 \%\left(R_{\text {free }}=27.0 \%\right)$ at $2.3-\AA$ resolution (Table III). Currently 151 water molecules are included in the model.

As defined by PROCHECK (30), 90.0\% of the residues lie within the most favored region of the Ramachandran plot. Three residues fall within disallowed regions. Of these, Ala-155 and Lys-219 both lie within loop regions with weak density, whereas Ile-212 is a hydrophobic core residue with excellent density. Residues with the highest $\mathrm{B}$ factors are located exclusively at the termini or in surface-exposed loops. Certain parts of the molecule have been omitted from the model where they correspond to regions of poor electron density, likely due to disorder. These are residues $1-3$ at the $\mathrm{N}$ terminus, seven residues at the $\mathrm{C}$ terminus (six of which are non-native and result from the genetic construct), and an external loop comprising residues 74-90. The apparent flexibility of these latter residues is consistent with the increased susceptibility of the G105D mutant enzyme to proteolysis compared with PBP 5 wild type. Proteolysis of sPBP 5' generates characteristic 30and $10-\mathrm{kDa}$ fragments by cleavage between residues 87 and 88 , which reside within this external loop (31).

\section{Structure Description}

sPBP $5^{\prime}$ is composed of two domains that are oriented approximately at right angles to each other (Fig. 2). Each domain is formed from contiguous primary sequence, residues 3 to 262 for domain 1 and residues 263 to 356 for domain 2 . The $\mathrm{N}$ terminal region of sPBP $5^{\prime}$ is a loop that extends away from domain 1 and makes a slight contact with domain 2 via Lys-6.

Domain 1-The principal feature of domain 1 is a fivestranded anti-parallel $\beta$ sheet that forms the hydrophobic core. This is packed on one side by an extended loop at the $\mathrm{N}$ terminus, $\alpha 10$, and a loop connecting $\beta 8$ and $\beta 9$ that contains a small $\alpha$ helix $(\alpha 9)$. The other side is comprised primarily of an array of seven $\alpha$ helices. In addition, there are three $\beta$ hairpinlike structures, $\beta 3-\beta 4, \beta 5-\beta 6$, and $\beta 7-\beta 8$, one of which $(\beta 3-\beta 4)$ contains the disordered residues $74-90$. The active site of PBP 
TABLE III

Statistics of the final model

$\begin{array}{lc}\text { Resolution range }(\AA) & 15.0-2.3 \\ \text { Sigma cut-off applied } & 0.0 \\ \text { Number of reflections used in refinement } & 17,208 \\ \text { Percentage of reflections used in } R_{\mathrm{FREE}} & 10.0 \\ \text { Completeness of data in resolution range }(\%) & 95.4 \\ \text { Number of protein atoms } & 2,597 \\ \text { Number of water molecules } & 151 \\ R \text { factor }(\%) & 19.6 \\ \text { Free } R \text { factor (\%) } & 27.0 \\ \text { r.m.s. deviations from ideal stereochemistry } & \\ \text { Bond lengths }(\AA) & 0.011 \\ \text { Bond angles }\left({ }^{\circ}\right) & 2.936 \\ \text { Dihedrals }\left({ }^{\circ}\right) & 24.73 \\ \left.\text { Impropers ( }{ }^{\circ}\right) & 2.552 \\ \text { Mean B factor (main chain) }\left(\AA^{3}\right) & 39.29 \\ \text { r.m.s. deviation in main chain B factor }\left(\AA^{3}\right) & 1.36 \\ \text { Mean B factor (side chains and waters) }\left(\AA^{3}\right) & 39.18 \\ \text { r.m.s. deviation in side chain B factors }\left(\AA^{3}\right) & 1.55 \\ \text { Ramachandran plot } & \\ \text { Residues in most-favored region }(\%) & 90.0 \\ \text { Residues in additionally allowed regions }(\%) & 8.3 \\ \text { Residues in generously allowed regions }(\%) & 0.7 \\ \text { Residues in disallowed regions }(\%) & 0.1 \\ \end{array}$

5 is located within this domain at the boundary of the fivestranded $\beta$-sheet and the helical array (see below). It is immediately apparent that domain 1 has the same fold as Class A and Class C $\beta$-lactamases (discussed below).

Domain 2-This domain, which is almost exclusively comprised of $\beta$ structure, is a sandwich of two anti-parallel $\beta$ sheets, one three-stranded and the other two-stranded. The two-stranded sheet is kinked at residues Gly-272 and Asp-293, causing the two sides of the sheet to be at right angles. The effect of this is to form a loose $\beta$ barrel at the $\mathrm{C}$-terminal end of the domain. Compared with domain 1, domain 2 has a relatively hydrophobic surface as judged by an electrostatic plot (data not shown), although none of these hydrophobic residues are particularly well conserved in other CPases. The significance of this apparent hydrophobicity and the role of this domain in the function of the protein are unknown. A search of the Protein Data Bank using the DALI server (32) revealed no genuine structural similarities to domain 2 .

Domain Interface-Since the hydrophobic cores of domains 1 and 2 extend to include the domain interface and because this region has comparatively low $\mathrm{B}$ factors, significant movements between domains 1 and 2 seem unlikely, suggesting that the relative juxtaposition of the two domains is conserved.

\section{Sequence Alignment to Other CPases and Secondary Structure Assignment}

A Blast search of the GenBank ${ }^{\mathrm{TM}}$ data base with $E$. coli PBP 5 as a query sequence identified two other $E$. coli PBPs (PBP 6 and PBP 6b) and PBPs from Salmonella typhimurium and Haemophilus influenzae as its closest matches. Alignment of these PBPs is shown in Fig. 3 along with secondary structure assignments from the three-dimensional structure of $\mathrm{SPBP} 5^{\prime}$. As expected, the regions of highest identity reside in and around the conserved sequence motifs (shown in parentheses) associated with the active site in domain 1 : residues $42-53$ $\left(\mathrm{Ser}^{44}-X-X\right.$-Lys $\left.{ }^{47}\right)$, residues $110-114\left(\mathrm{Ser}^{110}-X\right.$-Asn $\left.{ }^{112}\right)$, and residues $210-217\left(\mathrm{Lys}^{213}-\mathrm{Thr}^{214}-\mathrm{Gly}^{215}\right.$ ) (19). Two additional highly conserved regions, comprising residues $149-154$ and 195-203, also are located in close proximity to the active site (see Fig. 4). In general, the residues in domain 2 are less conserved than domain 1 , and most of the conserved residues are within the hydrophobic core.

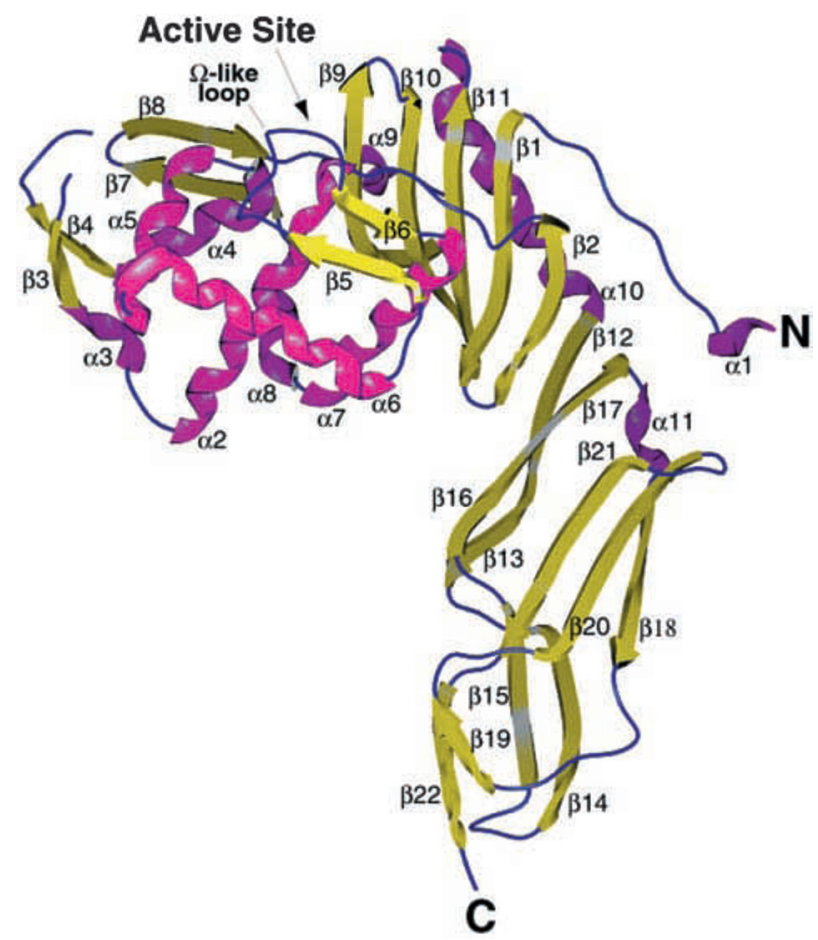

FIG. 2. The structure of $\mathbf{s P B P} \mathbf{5}^{\prime}$. The structure of sPBP $5^{\prime}$ with secondary structure assignments and the location of $\mathrm{N}$ and $\mathrm{C}$ termini are shown. In this view, the cytoplasmic membrane is at the bottom of the molecule, and the entrance to the active site is at the top. This figure was produced with the program SPOCK (available on the World Wide Web).

\section{Active Site}

The location of the active site is readily identified by plotting the conserved sequence motifs onto the SPBP $5^{\prime}$ structure. In common with other PBPs and $\beta$-lactamases, the active site is located in the cleft between the five-stranded anti-parallel $\beta$ sheet and the large $\alpha$ helical cluster. As viewed in Fig. 2, the entrance to the active site is at the opposite end of the molecule from the $\mathrm{C}$ terminus and the membrane anchor (which is missing in our construct), in an ideal position to interact with cell wall peptides. The architecture of active site (Fig. 4) is comprised of the following elements: helix $\alpha 2$ (yellow), containing Ser- 44 and Lys- 47 of the SXXK tetrad; the turn between helices $\alpha 4$ and $\alpha 5$ (green), containing Ser-110 and Asn-112 of the SXN triad; and $\beta 9$ (orange), the edge strand of the $\beta$ sheet containing Lys-213, Thr-214, and Gly-215 of the KT(S)G triad. In addition, the loops between $\beta 5$ and $\beta 6$ and between $\beta 8$ and $\alpha 9$ also contribute residues to the active site. These include His-151, present on the extended loop (blue) at the bottom of the cavity, and Arg-198, located on the loop (purple) at the top of the cavity. Both of these latter residues are conserved in related CPases (Fig. 4). The identification of Ser-44, Lys-47, Ser-110, Asn-112, and Lys-213 as active site residues is consistent with data showing that mutation of any of these renders PBP 5 highly deficient or, in some cases, completely inactive in both antibiotic binding and carboxypeptidation $(19,33)$.

The hydrogen-bonding network within the active site is extensive (Fig. 4). The $\epsilon-\mathrm{NH}_{2}$ group of Lys-47 plays a central role in this network, forming hydrogen bonds with the hydroxyl group of Ser-44, the amide carbonyl group of Asn-112, and the backbone carbonyl groups from both Ser-110 and His-151. In common with other penicillin-interacting enzymes, the $\epsilon-\mathrm{NH}_{2}$ group of Lys-213 forms a hydrogen bond with Ser-110. In contrast to Class A $\beta$-lactamases, in which two structurally conserved water molecules are typically observed within the 


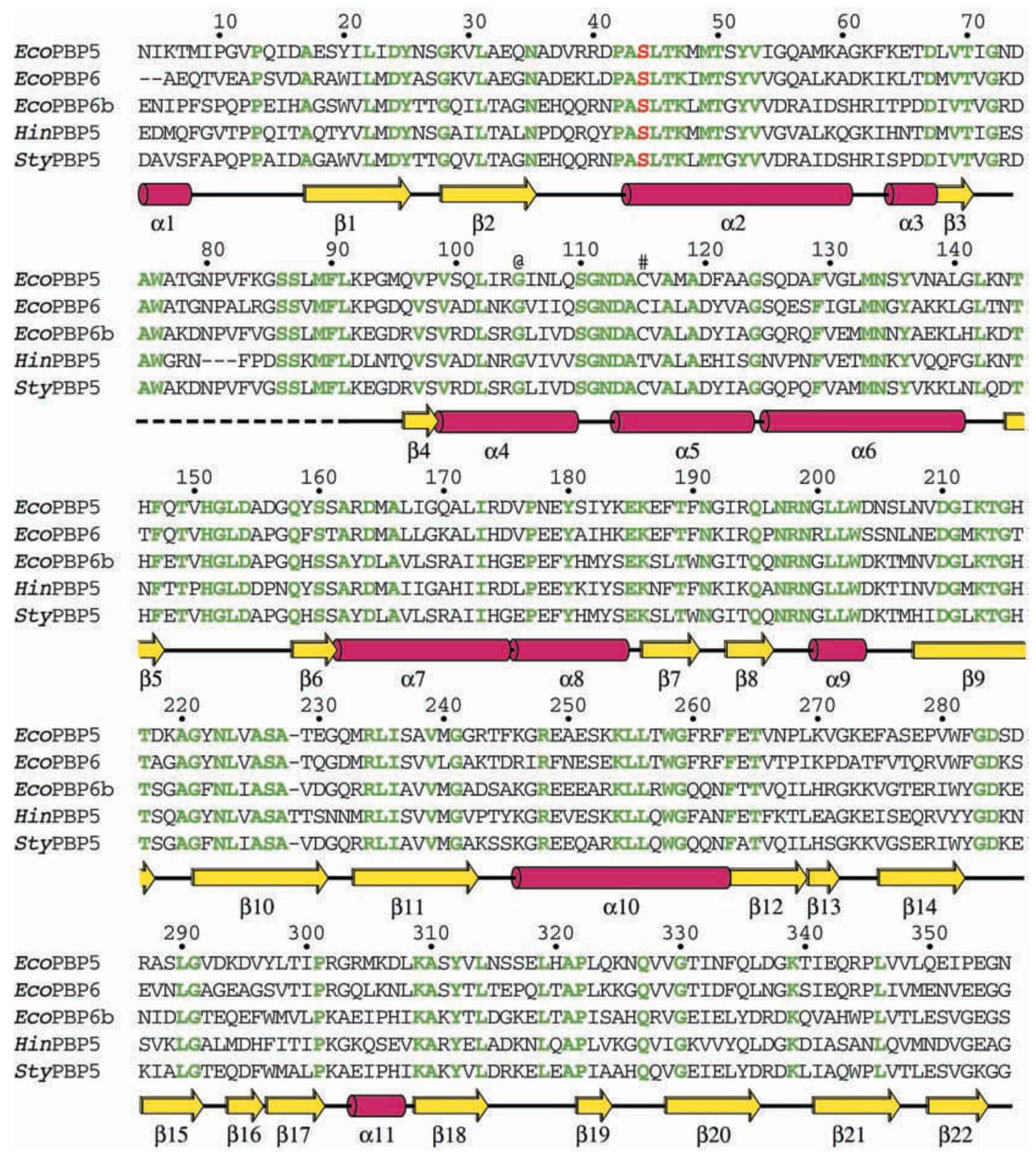

FIG. 3. Sequence alignment and secondary structure assignment. Alignment of the soluble, mature form of $E$. coli PBP 5 (Swiss Protein Database accession P04287) with E. coli PBP 6 (P08506) and PBP 6b (P33013), H. influenzae PBP 5 (P44466), and S. typhimurium PBP 6b (P37604). Conserved residues among all five PBPs are depicted in green. The active site serine is shown in red, the site of the mutation in sPBP $5^{\prime}$ is marked with by a @, and the cysteine residue in PBP 5 that reacts with sulfhydryl reagents is marked by a (\#). The secondary structure assignments are shown below. The dashed line represents the disordered region 74-90. The first three residues and the last six residues (the latter of which are non-native and a result of the genetic construct) of sPBP $5^{\prime}$ have been omitted for clarity.

hydrogen bonding network (34), there are no visible water molecules in the immediate vicinity of the active site. However, further refinement and higher resolution data of SPBP 5 ' may be necessary to firmly establish the presence or absence of potential active site water molecules.

\section{Structural Homology of sPBP 5' and TEM-1 $\beta$-Lactamase}

One of the most outstanding features of the sPBP $5^{\prime}$ structure is the similarity of domain 1 with the fold of Class A $\beta$-lactamases, as represented by TEM-1 (35) and PC1 (36). Notably, this structural similarity is considerably more pronounced than previously reported PBP structures, namely the Streptomyces R61 D,D-peptidase (37) and PBP 2x from Streptococcus pneumoniae (38). The main chain atoms in the 209 residues of domain 1 of SPBP $5^{\prime}$ composing the common elements of secondary structure of penicillin-interacting proteins can be superimposed onto the PC1 $\beta$-lactamase fold (36) with an r.m.s. deviation of $2.7 \AA$ and the TEM-1 $\beta$-lactamase (39) fold with an r.m.s. deviation of $2.5 \AA$.

The similarity of domain 1 of sPBP $5^{\prime}$ and TEM- $1 \beta$-lactamase is shown in Fig. 5. The most significant differences between the two structures are 1) the N-terminal helix of TEM-1 is replaced by an extended chain in $\left.\mathrm{SPBP} 5^{\prime}, 2\right)$ the disordered region between residues 74 and 90 in sPBP $5^{\prime}$ is visible in TEM-1 as a small helix with connecting loops, 3 ) the extended loop (colored blue in Fig. 4, of sPBP $5^{\prime}$ has a slightly different conformation compared with its counterpart in TEM-1, the so-called $\Omega$ loop, and 4) in sPBP $5^{\prime}$ the connection between helices $\alpha 8$ and $\alpha 9$ contains a large $\beta$ hairpin, whereas the equivalent connection in TEM- 1 , between helices $\alpha 9$ and $\alpha 10$, is direct.

A comparable degree of similarity with Class A $\beta$-lactamases was also observed in the recently determined structure of the Streptomyces K15 D,D-transpeptidase (40). In fact, the K15 PBP 


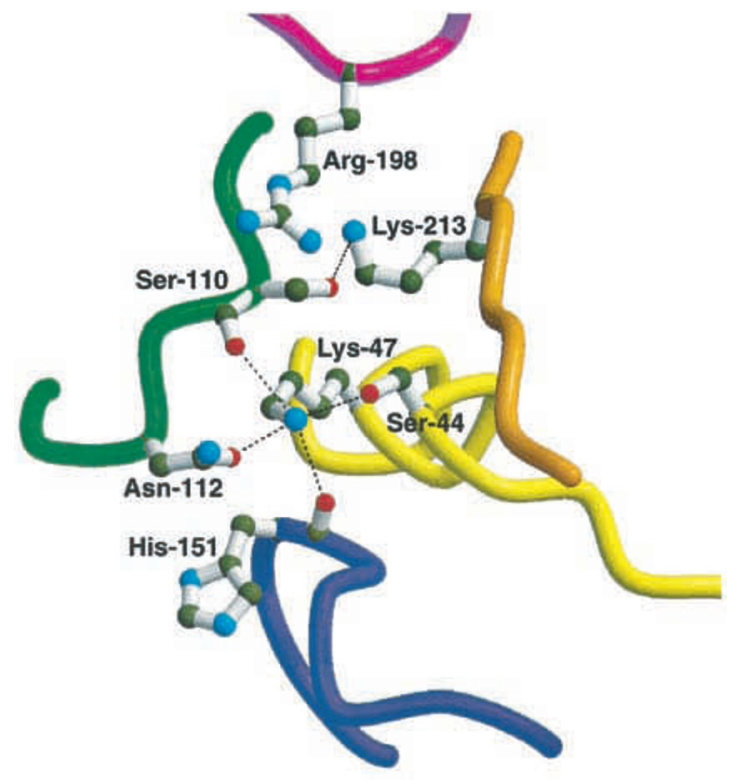

FIG. 4. Active site architecture of $\mathbf{s P B P} \mathbf{5}^{\prime}$. The active site is formed by five structural elements (colored yellow, green, blue, purple, and orange). Potential hydrogen bonds are denoted by the dashed lines. Note the orientations of the imidazole side chain and backbone carbonyl group of His-151 and the position of Arg-198. This figure was produced using MOLSCRIPT (51).

and $\mathrm{sPBP} 5^{\prime}$ are close structural relatives. Domain 1 of sPBP 5' can be superimposed onto the K15 PBP structure with an r.m.s. deviation of $1.2 \AA$ between common main chain atoms $(212$ residues). The main differences of note are that the part of the K15 PBP structure equivalent to the disordered region in $\mathrm{SPBP}$ $5^{\prime}$ (residues $74-90$ ) is ordered and that the $\beta 7-\beta 8$ hairpin is considerably longer in the K15 PBP structure $(\beta 2 \mathrm{c}-\beta 2 \mathrm{~d})$, generating a four-stranded $\beta$ sheet on the surface of the K15 PBP. Unlike most other PBPs, the K15 PBP does not contain a traditional hydrophobic transmembrane anchor. Thus, it has been proposed that the $\beta 2 \mathrm{c}-\beta 2 \mathrm{~d}$ hairpin promotes the association of the K15 PBP with the cell membrane (40), which may explain its longer length.

The active site residues of SPBP $5^{\prime}$ and TEM- $1 \beta$-lactamase were aligned by the program LSQKAB (26) (Fig. 6). Ser-44, Lys-47, Ser-110, Asn-112, and Lys-213 are all spatially conserved with the corresponding residues in TEM-1 $\beta$-lactamase, again emphasizing the extensive homology between these two proteins. Although the extended $\Omega$-like loop at the lower region of the active site of sPBP $5^{\prime}$ is similarly positioned to the $\Omega$ loop of TEM-1, there are significant conformational differences between the two. This is important because in Class A $\beta$-lactamases, the $\Omega$ loop contains two residues, Glu-166 and Asn-170, that are critical for the rapid hydrolysis of the acyl-enzyme complex $(41,42)$. By analogy, the $\Omega$-like loop in PBP 5 (residues 147-157) may also contain similar residues responsible for deacylation, thus explaining the high $\beta$-lactamase activity observed in wild-type PBP 5 (see "Discussion").

\section{DISCUSSION}

Here we report the structure of a soluble, mutant form of PBP 5' solved to 2.3-Å resolution by x-ray crystallography. The structure of sPBP $5^{\prime}$ reveals two domains, a penicillin-binding domain and a domain of unknown function. The fold of the penicillin-binding domain is highly similar to Class A $\beta$-lactamases, further emphasizing the close evolutionary relationship between these two classes of penicillin-interacting proteins.

Catalytic Function-PBP 5 is a serine-based D-alanine carboxypeptidase that is functionally similar to the well studied family of serine proteases. Although both PBP 5 and the serine proteases proceed through formation of an acyl-enzyme intermediate followed by deacylation of the acylated enzyme, they have evolved distinctly different catalytic mechanisms. The close structural similarity of SPBP 5 ' with Class A $\beta$-lactamases suggests a common mechanism of acylation by $\beta$-lactam antibiotics for the two classes of enzymes. In the light of the structure of PBP 5', the potential roles in catalysis of the conserved active site residues of PBP 5 can now be discussed.

Acylation of PBP 5 with $\beta$-Lactam Antibiotics and Peptide Substrates-The acylation reaction comprises four steps: formation of the non-covalent complex, nucleophilic attack, tetrahedral intermediate formation, and collapse to the acyl-enzyme. Mutational evidence has shown that, at least for $\beta$-lactam antibiotics, a positive charge at position 213 is crucial for formation of the acyl-enzyme complex (33), suggesting that Lys-213 (of the KTG triad) interacts with the carboxylate group on both $\beta$-lactam antibiotics and peptide substrates. Interestingly, the equivalent residue in $\beta$-lactamases, Lys-234, has been proposed to stabilize the transition state $(43,44)$. Since in our structure the $\epsilon$-amino group of Lys-213 interacts via potential hydrogen bonds with the hydroxyl group of Ser-110 and the carbonyl group of Asn-107, an alternative role of this residue may be simply to maintain the correct active site architecture rather than to interact with substrate directly. It is interesting to note that mutation of Lys-213 to arginine has no effect on penicillin binding and hydrolysis yet abolishes CPase activity (33). Such a mutation would increase the distance between two key components of the active site, $\beta 9$ and the $\mathrm{C}$-terminal end of $\alpha 4$, by approximately $1 \AA$. This may lower the binding affinity for peptide substrates, which presumably have a larger binding footprint without affecting the smaller binding site for penicillin.

Acylation starts by nucleophilic attack of the active site Ser-44 (22) on the amide bond of the peptide substrate or $\beta$-lactam ring. In serine proteases, the nucleophilicity of the serine residue is activated by the so-called "charge-relay" system involving a histidine and aspartic acid residue (45). In our structure it is difficult to assess which of the surrounding residues enhance the nucleophilicity of Ser-44. One possibility is that the amino group of Lys-47, which is within hydrogenbonding distance of the Ser-44 hydroxyl, acts as a general base. For this to occur, however, the active site environment must promote the deprotonated state of the $\epsilon-\mathrm{NH}_{2}$ group, requiring a reduction in its $\mathrm{p} K_{a}$ of approximately $3 \mathrm{pH}$ units. This might be achieved via its hydrogen-bonding interactions with Asn-112 and the carbonyl groups of His-151 and Ser-110 (Fig. 4). An alternative possibility is that the nucleophilicity of Ser-44 may be enhanced by the dipole moment of the helix $\alpha 2$. The role of the helix dipole has recently been suggested to increase the nucleophilicity of the active site cysteine in $\beta$-ketoacyl-acyl carrier protein synthase III, a bacterial enzyme that catalyzes a Claisen condensation in type II fatty acid synthesis (46).

It is interestingly to note that one characterized mutation, D175N, which abolishes CPase activity and decreases acylation by 50 -fold (19), probably acts indirectly by perturbing the precise arrangement of the active site rather than having a direct role in catalysis as previously suggested. Our structure shows that the effect of this mutation is to break a hydrogen bond between the side chains of Asp-175 and Tyr-52, located at the C-terminal end of helix $\alpha 2$, more than $20 \AA$ from Ser-44. That such a subtle change has such a large impact on activity is evidence that the precise positioning of the N-terminal end of $\alpha 2$, which contains Ser-44 and Lys-47, is critical for catalysis.

The developing negative charge on the carbonyl oxygen of the tetrahedral intermediate is likely stabilized by an oxyanion 


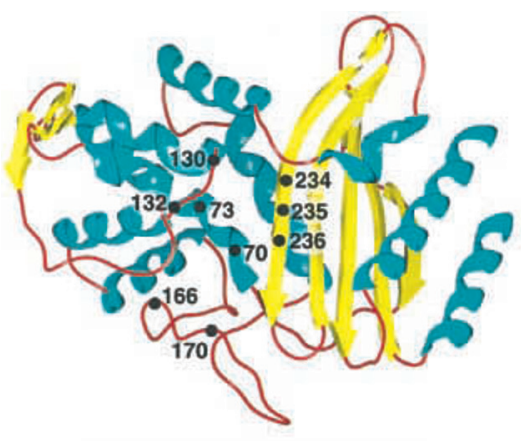

TEM-1 $\beta$-lactamase

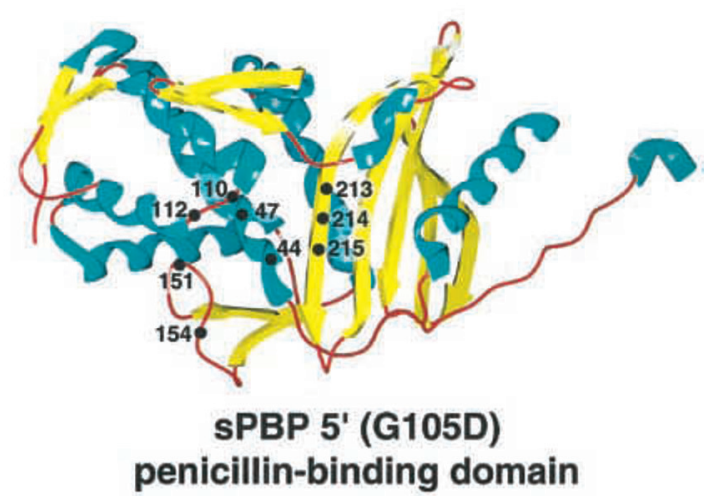

penicillin-binding domain

FIG. 5. Structural homology of TEM-1 $\boldsymbol{\beta}$-lactamase and the penicillin-binding domain of $\mathbf{s P B P} \mathbf{5}^{\prime}$. The view of $\mathrm{sPBP} 5^{\prime}$ is similar to that shown in Fig. 4. The penicillin-binding domain of sPBP $5^{\prime}$ is comprised of residues 1-265. The positions of active site residues are shown by a along with their residue numbers. This figure was produced using the program SPOCK (available on the World Wide Web).

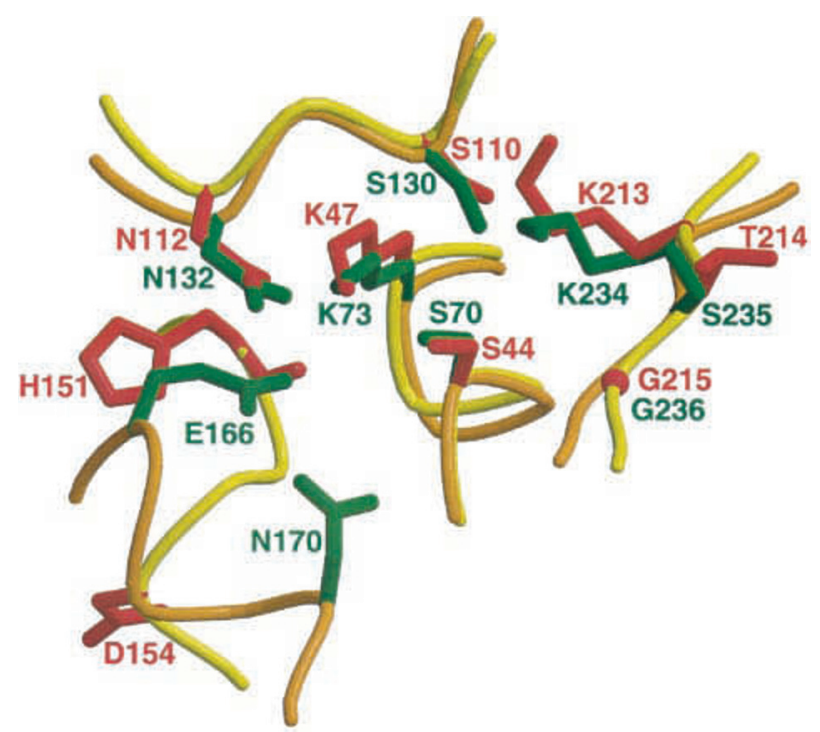

FIG. 6. Alignment of the active site residues of $\mathbf{S P B P} 5^{\prime}$ and TEM-1 $\beta$-lactamase. The active site residues of sPBP $5^{\prime}$ and TEM-1 $\beta$-lactamase (39) were aligned with the program LSQKAB (26). Backbone residues from sPBP $5^{\prime}$ are depicted in orange, and active site amino acids are shown in red. In TEM-1 $\beta$-lactamase, backbone residues are yellow, and active site amino acids are green. The carbonyl group of His-151 is also shown. This figure was produced using MOLSCRIPT (51).

hole similar to that proposed for many other enzymes, including serine proteases, $\beta$-lactamases, and other PBPs. In PBP 5 the oxyanion hole is potentially formed by backbone amide groups of residues 214 and 216 from $\beta 9$ and Ser-44 on $\alpha 2$. Arg-198 is positioned above the active site and may also help stabilize the intermediate.

Hydrolysis of the Acyl-Enzyme Complex-The close structural overlap of PBP $5^{\prime}$ with Class A $\beta$-lactamases suggests that the relatively high deacylation rate of the $\beta$-lactam-bound PBP 5 may be mediated by the loop equivalent to the $\Omega$ loop in Class A $\beta$-lactamases. In the latter, two residues on this loop, Glu-166 and Asn-170, are intimately involved in the rapid deacylation of the acyl-enzyme complex $(42,47)$. In particular, Glu-166 is believed to activate a conserved water molecule that attacks the carbonyl of the acyl-enzyme $(41,48)$. By analogy, residues in the $\Omega$-like loop of PBP 5 may also position a catalytic water molecule that attacks the acyl-enzyme complex. Candidate residues from the $\Omega$-like loop include His-151 and Asp-154, both of which are conserved in other CPases (Fig. 3) and one of which, His-151, is close to the active site (Fig. 4). However, in our structure of the deacylation-defective PBP 5', neither of these side chains is sufficiently close to the active site to interact directly with substrate or potential catalytic water molecules (Fig. 4). An alternative mechanism for hydrolysis of the acyl-enzyme complex is that the backbone carbonyl moiety of His-151, which in our structure is hydrogen-bonded to Lys-47 (Fig. 4), participates in deacylation simply by favorably orienting a hydrolytic water molecule. This is supported by the spatial equivalence of carbonyl group of His-151 in PBP 5' with the carboxyl group of Glu-166 in TEM-1 (Fig. 6).

Further evidence that the $\Omega$-like loop in PBP $5^{\prime}$ may indeed be involved in deacylation comes from studies of deacylationdefective forms of PBP 5. In the G105D mutant, Gly-105 (small) is replaced by aspartate (charged and bulky), which may disrupt the packing of helix $\alpha 4$ with the adjacent 74-90 loop and cause the apparent flexibility of the latter observed in our crystal structure. In both Class A $\beta$-lactamases and the K15 $\mathrm{D}, \mathrm{D}$-transpeptidase, the equivalent loop is ordered and packs against the counterparts of helices $\alpha 4, \alpha 5$, and the $\Omega$-like loop in $\mathrm{SPBP} 5^{\prime}$. A second deacylation-defective PBP 5 is obtained through modification of Cys-115 by para-chloromercuribenzoate in the wild-type enzyme (18). This residue is located on helix $\alpha 5$ and packs into the space between $\alpha 2$ and $\alpha 4$. Introduction of such a bulky group would doubtless perturb this close packing and may also affect the conformation of the adjacent 74-90 loop. Thus it appears that a decrease in deacylation may result from changes in the specific region of the structure containing $\alpha 4, \alpha 5$, the 74-90 loop, and the $\Omega$-like loop. In the structure of wild-type PBP 5 , it will be interesting to note whether the 74-90 loop is more ordered and indeed whether the conformation of the $\Omega$-like loop is altered as a result.

Relationship of sPBP $5^{\prime}$ to $\beta$-Lactamases-The structural homology between the Streptomyces R61 D,D-peptidase and PC1 $\beta$-lactamase was noted as early as $1986(11,12)$, but the similarity between sPBP $5^{\prime}$ and TEM- $1 \beta$-lactamase is even more pronounced. A comparable degree of similarity to Class A $\beta$-lactamases also has been observed with the Streptomyces K15 D,D-transpeptidase (40). It has been suggested that each of the different classes of $\beta$-lactamases have evolved from various classes of PBPs (49). Our data are entirely consistent with this hypothesis.

The main structural difference between the penicillin binding domain of $\operatorname{sPBP} 5^{\prime}$ and Class A $\beta$-lactamases is in the sequence and conformation of the $\Omega$ loop, perhaps suggesting that Class A $\beta$-lactamases evolved directly from a PBP 5-like CPase. This would be logical because CPases already had a relatively efficient hydrolysis mechanism for peptide substrates. By incorporating two new amino acids on the $\Omega$ loop and by removing residues specific for CPase activity, $\beta$-lacta- 
mases evolved a highly efficient hydrolysis mechanism and at the same time altered their specificity toward $\beta$-lactam antibiotics and away from peptide substrates.

Role of Domain 2-The two-domain structure of PBP 5, in which catalytic activity resides in domain 1 , is consistent with previous genetic studies showing that deletion of the protein after residue 263 results in an enzyme with near wild-type CPase activity and $\beta$-lactam antibiotic affinity (50). This, however, leaves the role of domain 2 unclear. One possibility is that this domain mediates interactions with other cell wall-synthesizing enzymes to recruit PBP 5 to areas of active cell wall synthesis. Indeed, the all $\beta$ structure of this domain may facilitate such interactions through hydrogen bonding across $\beta$ strands. In $E$. coli, the lytic transglycosylase, MltA, interacts with several PBPs as well as the scaffolding protein, MipA (8), suggesting the existence of a cell wall-synthesizing complex, of which PBP 5 may be a part. Alternatively, domain 2 may function simply as a linker to position the active site in domain 1 closer to the peptidoglycan layer, where it can interact with cell wall peptides.

Conclusion-By solving the crystal structure of a deacylation-defective mutant, we have obtained the first structural information for PBP 5. PBP 5 shares the same fold and has a common active site architecture with the well studied Class A $\beta$-lactamases. Inspection of this structure suggests that Lys-47 may act as the general base to promote the nucleophilicity of Ser-44 during acylation. In contrast, the mechanism of deacylation is less clear. However, solving the wild-type PBP 5 structure and comparing it to $\mathrm{PBP} 5^{\prime}$ should provide significant insight into how PBP 5 catalyzes hydrolysis of the acyl-enzyme complex. These studies are in progress.

\section{REFERENCES}

1. Ghuysen, J. M. (1988) Rev. Infect. Dis. 10, 726-732

2. Tipper, D. J., and Strominger, J. L. (1965) Proc. Natl. Acad. Sci. U. S. A. 54 1133-1141

3. Ghuysen, J. M. (1991) Annu. Rev. Microbiol. 45, 37-67

4. Spratt, B. G. (1975) Proc. Natl. Acad. Sci. U. S. A. 72, 2999-3003

5. Matsuhashi, M., Tamaki, S., Curtis, S. J., and Strominger, J. L. (1979) J. Bacteriol. 137, 644-647

6. Amanuma, H., and Strominger, J. L. (1980) J. Biol. Chem 255, 11173-11180

7. Denome, S. A., Elf, P. K., Henderson, T. A., Nelson, D. E., and Young, K. D. (1999) J. Bacteriol. 181, 3981-3993

8. Nelson, D. E., and Young, K. D. (2000) J. Bacteriol. 182, 1714-1721

9. Bush, K., and Sykes, R. B. (1984) in Antimicrobial Drug Resistance, pp. 1-31, Academic Press, Inc., New York, NY

10. Joris, B., Ghuysen, J.-M., Dive, G., Renard, A., Dideberg, O., Charlier, P., Frere, J.-M., Kelly, J. A., Boyington, J. C., Moews, P. C., and Knox, J. R. (1988) Biochem. J. 250, 313-324

11. Samraoui, B., Sutton, B. J., Todd, R. J., Artymiuk, P. J., Waley, S. G., and Phillips, D. C. (1986) Nature 320, 378-380

12. Kelly, J. A., Dideberg, O., Charlier, P., Wery, J. P., Libert, M., Moews, P. C.,
Knox, J. R., Duez, C., Fraipont, C., Joris, B., Dusart, J., Frere, J.-M., and Ghuysen, J.-M. (1986) Science 231, 1429-1431

13. Ghuysen, J.-M., and Dive, G. (1994) in Bacterial Cell Walls, pp. 103-129, Elsevier Science Publishers B. V., Amsterdam

14. Pratt, R. F., and Govardhan, C. P. (1984) Proc. Natl. Acad. Sci. U. S. A. 81, 1302-1306

15. Martin, M. T., and Waley, S. G. (1988) Biochem. J. 254, 923-925

16. Amanuma, H., and Strominger, J. L. (1984) J. Biol. Chem. 259, 1294-1298

17. Nicholas, R. A., and Strominger, J. L. (1988) J. Biol. Chem. 263, 2034-2040

18. Curtis, S. J., and Strominger, J. L. (1978) J. Biol. Chem. 253, 2584-2588

19. vanderLinden, M. P., deHaan, L., Dideberg, O., and Keck, W. (1994) Biochem. J. 303, 357-362

20. Pratt, J. M., Jackson, M. E., and Holland, I. B. (1986) EMBO J. 5, 2399-2405

21. Kustu, S. G., and Ames, G. F. L. (1974) J. Biol. Chem. 249, 6976-6983

22. Nicholas, R. A., Ishino, F., Park, W., Matsuhashi, M., and Strominger, J. L. (1985) J. Biol. Chem. 260, 6394-6397

23. Nicholas, R. A., and Strominger, J. L. (1988) Rev. Infect. Dis. 10, 733-738

24. Otwinowski, Z., and Minor, W. (1997) Methods Enzymol. 276, 307-326

25. Frere, J. M., Ghuysen, J. M., and Perkins, H. R. (1975) Eur. J. Biochem. 57, 353-359

26. Collaborative Computation Project (1994) Acta Crystallogr. Sec. D 50, 760-763

27. deLaFortelle, E., and Bricogne, G. (1997) Methods Enzymol. 276, 472-494

28. Abrahams, J. P., and Leslie, A. G. W. (1996) Acta Crystallogr. Sec. D 52, 30-42

29. Jones, T. A., Zhou, J. Y., Cowan, S. W., and Kjeldgaard, M. (1991) Acta Crystallogr. Sec. A 47, 110-119

30. Laskowski, R. A., MacArthur, M. W., Moss, D. S., and Thorton, J. M. (1993) J. Appl. Crystallogr. 26, 283-291

31. vanderLinden, M. P., Mottl, H., and Keck, W. (1992) Eur. J. Biochem. 204, 197-202

32. Holm, L., and Sander, C. (1995) Trends Biochem. Sci. 20, 478-480

33. Malhotra, K. T., and Nicholas, R. A. (1992) J. Biol. Chem. 267, 11386-11391

34. Matagne, A., Lamotte-Brasseur, J., and Frere, J. M. (1998) Biochem. J. 330, $581-598$

35. Rhazi, N., Galleni, M., Page, M. I., and Frere, J. M. (1999) Biochem. J. 341, $409-413$

36. Herzberg, O., and Moult, J. (1987) Science 236, 694-701

37. Kelly, J. A., Knox, J. R., Moews, P. C., Hite, G. J., Bartolone, J. B., Zhao, H. Joris, B., Frere, J. M., and Ghuysen, J. M. (1985) J. Biol. Chem. 260, 6449-6458

38. Pares, S., Mouz, N., Petillot, Y., Hakenbeck, R., and Dideberg, O. (1996) Nat Struct. Biol. 3, 284-289

39. Jelsch, C., Mourey, L., Masson, J. M., and Samama, J. P. (1993) Proteins 16, $364-383$

40. Fonzé, E., Vermeire, M., Nguyen-Distèche, M., Brasseur, R., and Charlier, P (1999) J. Biol. Chem 274, 21853-21860

41. Adachi, H., Ohta, T., and Matsuzawa, H. (1991) J. Biol. Chem. 266, 3186-3191

42. Lewis, E. R., Winterberg, K. M., and Fink, A. L. (1997) Proc. Natl. Acad. Sci. U.S. A. 94, $443-447$

43. Ellerby, L. M., Escobar, W. A., Fink, A. L., Mitchinson, C., and Wells, J. A. (1990) Biochemistry 29, 5797-5806

44. Brannigan, J., Matagne, A., Jacob, F., Damblon, C., Joris, B., Klein, D., Spratt B. G., and Frere, J. M. (1991) Biochem. J. 278, 673-678

45. Fersht, A. R. (1985) Enzyme Structure and Mechanism, 2nd Ed., pp. 406-407, W. H. Freeman and Co., New York

46. Davies, C., Heath, R. J., White, S. W., and Rock, C. O. (2000) Structure Fold. Des. 8, 185-195

47. Guillaume, G., Vanhove, M., Lamotte-Brasseur, J., Ledent, P., Jamin, M., Joris, B., and Frere, J. M. (1997) J. Biol. Chem. 272, 5438-5444

48. Strynadka, N. C., Adachi, H., Jensen, S. E., Johns, K., Sielecki, A., Betzel, C. Sutoh, K., and James, M. N. (1992) Nature 359, 700-705

49. Massova, I., and Mobashery, S. (1998) Antimicrob. Agents Chemother. 42, 1-17

50. vanderLinden, M. P., deHaan, L., and Keck, W. (1993) Biochem. J. 289, 593-598

51. Kraulis, P. J. (1991) J. Appl. Crystallogr. 24, 946-950 